The Astrophysical Journal, 669:1072-1084, 2007 November 10

(C) 2007. The American Astronomical Society. All rights reserved. Printed in U.S.A.

\title{
NEAR-INFRARED INTERFEROMETRIC, SPECTROSCOPIC, AND PHOTOMETRIC MONITORING OF T TAURI INNER DISKS
}

\author{
J. A. Eisner, ${ }^{1}$ L. A. Hillenbrand ${ }^{2}$ R. J. White, ${ }^{3}$ J. S. Bloom, ${ }^{1}$ R. L. Akeson, ${ }^{4}$ and C. H. Blake ${ }^{5}$ \\ Received 2007 May 28; accepted 2007 July 24
}

\begin{abstract}
We present high angular resolution observations with the Keck Interferometer, high-dispersion spectroscopic observations with Keck/NIRSPEC, and near-IR photometric observations from PAIRITEL of a sample of 11 solartype T Tauri stars in nine systems. We use these observations to probe the circumstellar material within 1 AU of these young stars, measuring the circumstellar-to-stellar flux ratios and angular size scales of the $2.2 \mu \mathrm{m}$ emission. Our sample spans a range of stellar luminosities and mass accretion rates, allowing investigation of potential correlations between inner disk properties and stellar or accretion properties. We suggest that the mechanism by which the dusty inner disk is truncated may depend on the accretion rate of the source; in objects with low accretion rates, the stellar magnetospheres may truncate the disks, while sublimation may truncate dusty disks around sources with higher accretion rates. We have also included in our sample objects that are known to be highly variable (based on previous photometric and spectroscopic observations), and for several sources, we obtained multiple epochs of spectroscopic and interferometric data, supplemented by near-IR photometric monitoring, to search for inner disk variability. While timevariable veilings and accretion rates are observed in some sources, no strong evidence for inner disk pulsation is found.

Subject headings: circumstellar matter -

stars: individual (AA Tau, BM And, CI Tau, DI Cep, DK Tau, RW Aur, V1002 Sco, V1331

Cyg) - stars: pre-main-sequence — techniques: high angular resolution -

techniques: interferometric
\end{abstract}

\section{INTRODUCTION}

Protoplanetary disks are an integral part of the star and planet formation process, and studying such disks around early analogs of stars like our own Sun can provide insight into how planetary systems form. T Tauri stars are $\lesssim 2 M_{\odot}$ pre-main-sequence objects, and their circumstellar environments are an ideal laboratory for studying the initial conditions of planet formation. A wealth of evidence, including direct imaging at millimeter and optical wavelengths (e.g., Koerner \& Sargent 1995; Dutrey et al. 1996; McCaughrean \& O’Dell 1996), and modeling of spectral energy distributions (SEDs; e.g., Adams et al. 1988; Bertout et al. 1988; Beckwith et al. 1990), has confirmed the long-espoused hypothesis that $\mathrm{T}$ Tauri stars are surrounded by substantial disks of dust and gas. Moreover, observed line profiles and UV continuum excesses indicate that $\mathrm{T}$ Tauri stars are accreting material from their circumstellar disks (e.g., Walker 1972; Basri \& Bertout 1989; Edwards et al. 1994; Gullbring et al. 1998).

Study of the innermost disk regions may reveal the mechanism by which material is accreted through the disk onto the star. In the current paradigm, T Tauri disks are truncated by the stellar magnetosphere within the corotation radius, with material accreting along magnetic field lines onto high-latitude regions of the star (e.g., Königl 1991; Shu et al. 1994). For typical T Tauri star masses, radii, magnetic field strengths, and accretion rates, predicted trun-

\footnotetext{
1 Department of Astronomy, University of California, 601 Campbell Hall, Berkeley, CA 94720.

2 Department of Astronomy, California Institute of Technology, MC 105-24, Pasadena, CA 91125.

3 Department of Physics, University of Alabama, 201B Optics Building, John Wright Drive, Huntsville, AL 35899.

4 Michelson Science Center, California Institute of Technology, MC 100-22, Pasadena, CA 91125.

5 Harvard College Observatory, Cambridge, MA 02138.
}

cation radii range from $\sim 0.02$ to $0.2 \mathrm{AU}$ (e.g., Johns-Krull 2007). Previous studies have shown that the dusty component of disks is generally truncated farther out than the magnetospheric radius (e.g., Eisner et al. 2005; Akeson et al. 2005b). Dust sublimation is caused by heating from the central star, plus possible additional heating due to viscous dissipation of accreting material and luminosity from the impact of the accretion flow onto the stellar photosphere. If the accretion luminosity varies with time, then the inner dust disk edge may pulsate as the sublimation radius moves in or out. Some evidence for such variability may be present in near-IR photometric monitoring data (e.g., Skrutskie et al. 1996; Carpenter et al. 2001; Eiroa et al. 2002). Below, we discuss a search for inner disk pulsations using multiple epochs of spatially resolved observations at $2.2 \mu \mathrm{m}$.

Knowledge of the spatial and temperature structure of protoplanetary disks at sub-AU radii is important for understanding the properties of dust and gas in regions where terrestrial planet formation occurs. Temperature is the crucial parameter in determining the radii where dust and water ice, key building blocks of terrestrial planets, can condense (e.g., Hayashi 1981). Spatial structure is important for understanding how the close-in extrasolar planets discovered by radial velocity surveys (e.g., Marcy et al. 2005 ) either formed at, or migrated to, their observed orbital radii (e.g., Lin et al. 1996).

Infrared interferometric observations are necessary to spatially resolve disk regions within $\sim 1$ AU of the central stars. Such spatially resolved observations are the only way to probe directly the geometry and temperature of these inner disk regions. The Keck Interferometer has recently enabled spatially resolved observations of protoplanetary disks around several approximately solartype T Tauri stars (Colavita et al. 2003; Eisner et al. 2005, 2006; Akeson et al. 2005a); these observations constrained the dust disk inner radius and temperature, and showed evidence that the inner disk gas extends farther in toward the central star. Moreover, when 
these data are combined with previous spatially resolved observations of more massive, luminous objects (Millan-Gabet et al. 1999, 2001; Eisner et al. 2003, 2004, 2007; Leinert et al. 2004; van Boekel et al. 2004; Monnier et al. 2005, 2006; Akeson et al. 2005b; Liu et al. 2007), the inner disk properties as a function of luminosity can be investigated (e.g., Millan-Gabet et al. 2007). In general, these observations are consistent with expectations for disks with directly illuminated inner walls; however, there are notable exceptions for solar and subsolar mass T Tauri stars, motivating further observations.

Here we present near-IR interferometric observations of a sample of low-mass (including solar and subsolar mass) T Tauri stars. These observations spatially resolve the near-IR emission and allow measurements of the size of the emitting region. In addition, we use spectroscopic and photometric measurements to constrain the relative stellar and circumstellar contributions to the near-IR emission, as well as the accretion luminosities of our targets. All of these data constrain the inner disk properties of our sample, and in cases where multiple epochs of data were obtained, enable study of inner disk variability.

\section{OBSERVATIONS}

\subsection{Keck Interferometer Observations}

We used the Keck Interferometer (KI) to obtain spatially resolved measurements of the near-IR emission from a sample of $11 \mathrm{~T}$ Tauri stars (Table 1). In addition, we incorporate into our data set a measurement of RW Aur A obtained on 2002 October 23 by Akeson et al. (2005a). Among the objects for which we have multiple epochs of data, DK Tau A, AA Tau, and RW Aur A are known to be photometrically variable at optical through nearIR wavelengths (Herbst et al. 1994; Skrutskie et al. 1996; Eiroa et al. 2002).

$\mathrm{KI}$ is a fringe-tracking, long-baseline near-IR Michelson interferometer that combines the light from the two $10 \mathrm{~m}$ Keck apertures over an $85 \mathrm{~m}$ baseline (Colavita \& Wizinowich 2003; Colavita et al. 2003). For each target, we measured squared visibilities $\left(V^{2}\right)$ at $K$ band $\left(\lambda_{0}=2.2 \mu \mathrm{m}, \Delta \lambda=0.4 \mu \mathrm{m}\right)$. The system visibility (i.e., the point-source response of the interferometer) was measured using observations of unresolved calibrators (Table 1), weighted by the internal scatter in the calibrator and the temporal and angular proximity to the target source (Boden et al. 1998). Source and calibrator data were corrected for detection biases as described by Colavita (1999) and averaged into $5 \mathrm{~s}$ blocks. The calibrated $V^{2}$ for a target source is the average of the $5 \mathrm{~s}$ blocks in each integration, with uncertainties given by the quadrature addition of the internal scatter and the uncertainty in the system visibility. Systematic $V^{2}$ effects associated with enhanced performance of the adaptive optics system on one telescope relative to the other (which may depend on the brightness of the target) are calibrated by applying a "ratio correction" (e.g., Colavita 1999). Typical uncertainties are $\sim 5 \%$.

Measured $V^{2}$ are shown in Figure 1. The angular resolution of our observations is of order a milliarcsecond, which is sufficient to resolve the near-IR circumstellar emission, but not the central star. For expected stellar radii of $2-5 R_{\odot}$ and distances of $140-$ $700 \mathrm{pc}$ for our sample, the stars subtend $<0.2$ mas. Our measured $V^{2}$ thus include contributions from both the unresolved stellar component and the resolved circumstellar component.

\subsection{Keck/NIRSPEC Observations}

We obtained high-dispersion $K$-band spectra with NIRSPEC between 2004 November and 2006 July for our sample (Table 1).
We used the high-dispersion mode with the 3 pixel slit, which provides a resolving power of $R \sim 24,000$. The NIRSPEC-7 filter was used with an echelle position of 62.67 and a cross-disperser position of 35.51 . This provided seven spectral orders covering portions of the wavelength range between 1.99 and $2.39 \mu \mathrm{m}$. Included in these orders are various stellar atomic lines, the $\mathrm{CO}(2-0)$, $(3-1),(4-2)$, and (5-3) band heads, and the Brackett gamma $(\mathrm{Br} \gamma)$ line.

The spectra were calibrated and extracted using the "REDSPEC" package (e.g., McLean et al. 2003). Reduction included mapping of spatial distortions, spectral extraction, wavelength calibration, heliocentric radial velocity corrections, bias correction, flat fielding, and sky subtraction. We divided our target spectra by A0 V or A1 V stellar spectra (that were interpolated over broad $\mathrm{Br} \gamma \mathrm{ab}-$ sorption features) and multiplied by appropriate blackbody templates to calibrate the bandpass of the instrument and correct for telluric lines. For the two binary systems observed, which have separations of $2.3^{\prime \prime}$ (DK Tau AB) and 1.4" (RW Aur AB), the components are sufficiently well separated that their spectra could be extracted independently.

\subsection{PAIRITEL JHK Photometry}

We observed our sample with the $1.3 \mathrm{~m}$ Peters Automated Infrared Imaging Telescope (PAIRITEL; Bloom et al. 2006) and measured photometric fluxes at $J, H$, and $K_{s}$ bands. For each source, we obtained short exposures $(52 \mathrm{~ms})$ and relatively longer exposures (7.8 s). Short exposures were read out as "clearing exposures" before the longer exposures (this is the nominal process of double-correlated reads in infrared imaging). The short exposures provide unsaturated images of our targets, while the longer exposures yield high signal-to-noise ratio images of fainter stars within the observed fields that are used for photometric calibration. The camera provides $2^{\prime \prime}$ pixels over a $8.5^{\prime} \times 8.5^{\prime}$ field of view. Images are obtained simultaneously in $J, H$, and $K_{s}$ as part of the camera design.

Our data reduction consisted of background (sky + dark) subtraction (using dithered images to produce backgrounds) and flat fielding. Counts for target and calibrator objects were measured in apertures with 2 pixel radii, sky background was measured as the median value of pixels in an annulus spanning 10-20 pixels in radius, and the sky was subtracted from each pixel in the aperture. We calibrated the fluxes using measured Two Micron All Sky Survey (2MASS) magnitudes for other stars within the field of view of each target image.

Our photometric errors include scatter in measurements of source and calibrator counts, scatter in the magnitudes computed using different calibrators, and an assumed uncertainty in the 2MASS magnitudes of our calibrator stars of 0.017 mag. Measured fluxes and uncertainties for our sample are listed in Table 2.

Where possible, we selected calibrator stars that did not appear to vary from epoch to epoch; since no photometric standard stars were observed, our photometry is measured relative to calibrator stars within the observed fields. We evaluated calibrator variability by comparison of each calibrator to the mean value of all calibrators within an epoch; as we discovered variable sources, we excluded them from the calculated mean value and iterated. However, for some fields where only a few stars were visible, no nonvariable calibrator stars were found with this procedure (which may indicate unstable atmospheric or instrumental conditions); for these cases our photometric uncertainties include the apparent variability of the flux calibrators.

The binaries in our sample (DK Tau AB and RW Aur AB) are not resolved with the $2^{\prime \prime}$ pixels of the PAIRITEL camera. Thus, the 
TABLE 1

Target and Calibrator Properties and Observations

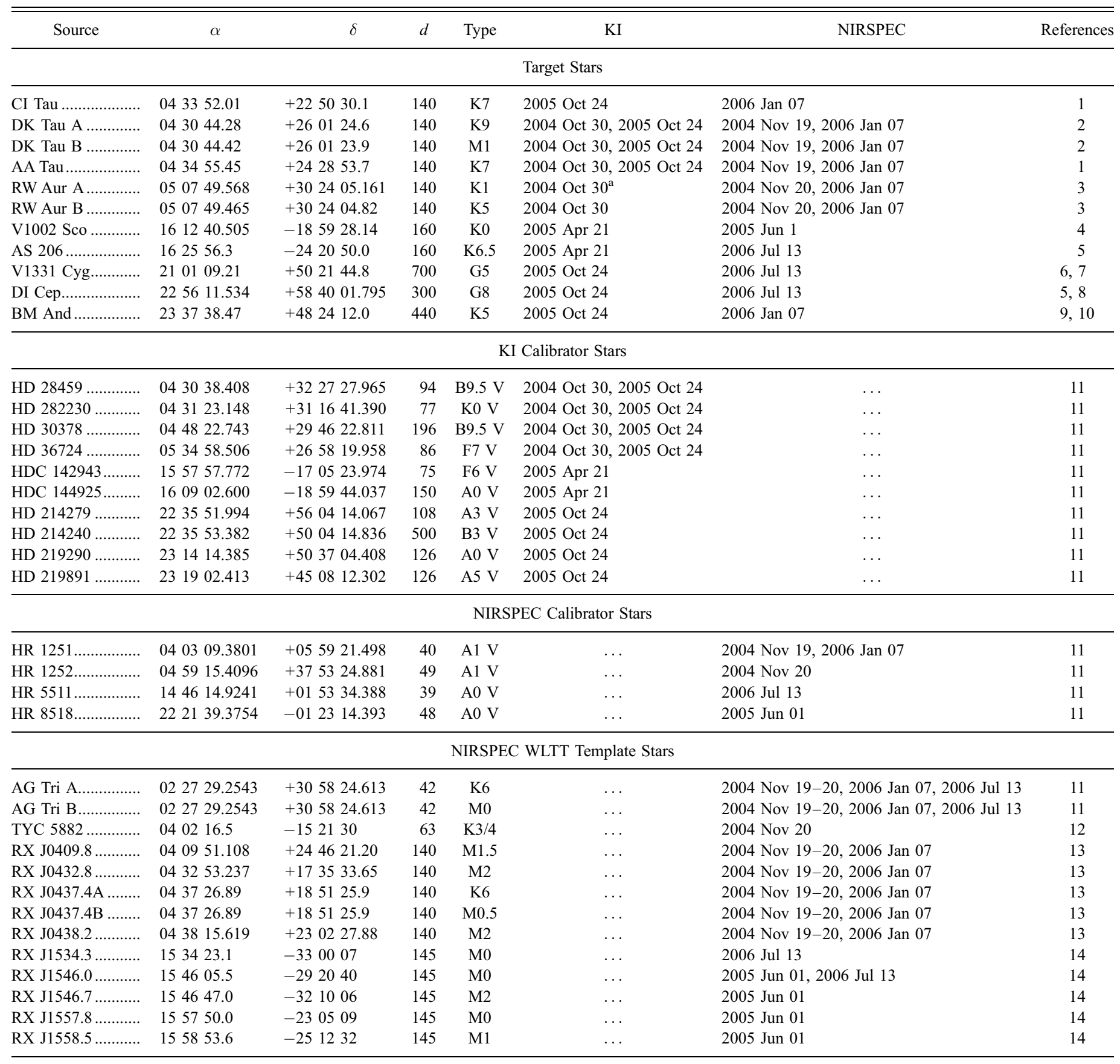

Notes.-All Taurus sources were assumed to be at a distance of $140 \mathrm{pc}$. Units of right ascension are hours, minutes, and seconds, and units of declination are degrees, arcminutes, and arcseconds.

${ }^{a}$ For RW Aur A, we also use KI measurements from 2002 October 23 (Akeson et al. 2005a).

References.-(1) Kenyon \& Hartmann 1995; (2) Monin et al. 1998; (3) White \& Ghez 2001; (4) Walter et al. 1994; (5) Herbig \& Bell 1988; (6) Chavarria-K. 1981; (7) Hamann \& Persson 1992; (8) Kholopov 1959; (9) Aveni \& Hunter 1969; (10) Mora et al. 2001; (11) Perryman et al. 1997; (12) Zuckerman \& Song 2004; (13) Wichmann et al. 2000; (14) Köhler et al. 2000.

photometry for these objects is the composite of both components. The $K$-band brightness ratios for DK Tau AB and RW Aur AB are 3.3 and 4.3, respectively (Simon et al. 1995; White \& Ghez 2001), and our photometry is thus dominated by the primary components.

\section{ANALYSIS}

In this section we use the data described in $\S 2$ to constrain the properties of the inner disk regions around our sample objects.
Our focus is on spatially resolving the circumstellar emission with our interferometry data. However, because we require measurements of the circumstellar-to-stellar flux ratio in order to properly model the circumstellar component of our $V^{2}$ measurements (e.g., Eisner et al. 2005), we begin by deriving this ratio from our NIRSPEC data ( $\S 3.1$ ). In $\S 3.2$ we model our spatially resolved interferometric measurements of the circumstellar emission from our targets with a simple geometric model. In $\S 3.3$ we derive 

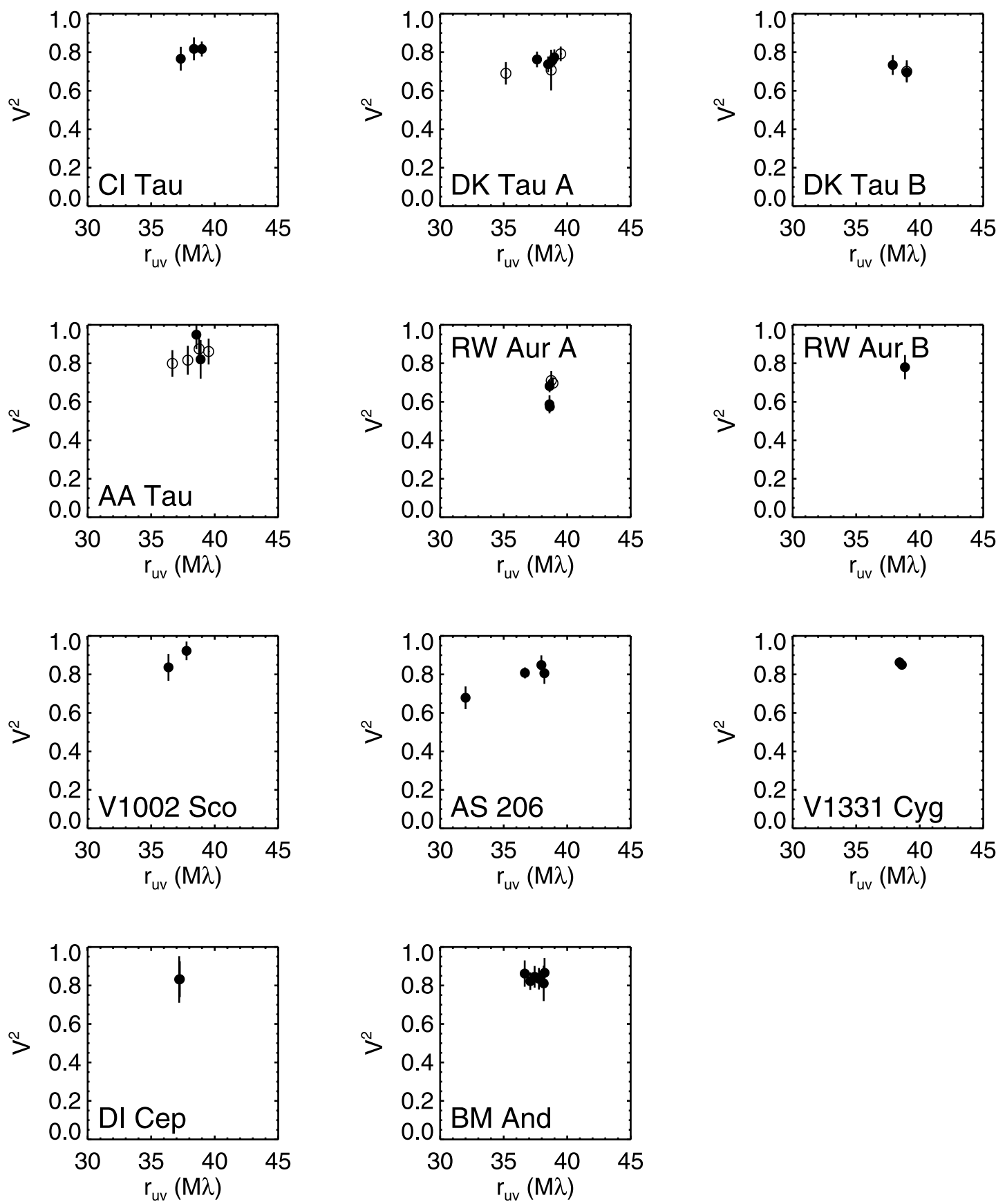

FIG. 1.-Measured $V^{2}$ from KI as a function of $u$ - $v$ radius, $r_{u v}$ ( $r_{u v}$ is equal to the projected baseline length divided by the wavelength). Given the angular resolution of

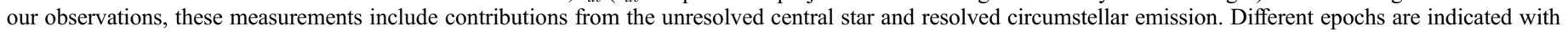
different symbols: open circles represent the second epoch (where available). The first epoch of KI data for RW Aur A are from Akeson et al. (2005a).

accretion luminosities for our targets to enable an investigation of how the inner disk geometries inferred from our interferometry data correlate with mass accretion rate.

\subsection{Circumstellar-to-Stellar Flux Ratios}

We use the NIRSPEC measurements described in $\S 2.2$ to derive $2 \mu \mathrm{m}$ veilings for our sample. The veiling is defined as the ratio of the circumstellar excess flux to the stellar flux in the $K$ band. Veilings are derived by comparing spectra of our target objects with spectra obtained (on the same night) for nonaccreting, weak-lined T Tauri stars (WLTTs). These WLTTs provide excellent templates, since they have stellar effective temperatures and surface gravities similar to the classical T Tauri stars in our sample, but show neither near-IR excess emission nor accretion signatures.

We select several atomic absorption lines for comparison between targets and templates. For a grid of WLTT templates, we perform a $\chi^{2}$ minimization between the lines seen in the target star and the same lines in the template stars after registration of the relative radial velocities, rotational broadening, and veiling. Radial velocity registration is accomplished via a cross-correlation of target and template star spectra. Rotational broadening is implemented by convolving the template spectrum with a Doppler line profile (Gray 1992). The template is veiled by adding a continuum 
TABLE 2

PAIRITEL $J H K_{\mathrm{S}}$ РнOTOMETRY

\begin{tabular}{|c|c|c|c|c|}
\hline Source & Date & $m_{J}$ & $m_{H}$ & $m_{K_{s}}$ \\
\hline \multirow[t]{2}{*}{ CI Tau ........................ } & 2005 Oct 24 & $8.50 \pm 0.02$ & $7.99 \pm 0.02$ & $7.51 \pm 0.03$ \\
\hline & 2005 Nov 20 & $8.25 \pm 0.02$ & $7.83 \pm 0.02$ & $7.34 \pm 0.02$ \\
\hline \multirow[t]{3}{*}{ DK Tau AB.......... } & 2004 Oct 18 & $7.94 \pm 0.02$ & $7.46 \pm 0.02$ & $7.03 \pm 0.03$ \\
\hline & 2004 Nov 05 & $7.95 \pm 0.02$ & $7.45 \pm 0.02$ & $6.98 \pm 0.02$ \\
\hline & 2005 Oct 24 & $7.83 \pm 0.05$ & $7.38 \pm 0.05$ & $6.92 \pm 0.08$ \\
\hline \multirow[t]{4}{*}{ AA Tau ..................... } & 2004 Oct 18 & $8.80 \pm 0.02$ & $8.37 \pm 0.02$ & $7.94 \pm 0.03$ \\
\hline & 2004 Nov 05 & $9.10 \pm 0.03$ & $8.58 \pm 0.03$ & $8.12 \pm 0.03$ \\
\hline & 2005 Oct 24 & $8.82 \pm 0.03$ & $8.38 \pm 0.03$ & $7.74 \pm 0.04$ \\
\hline & 2005 Nov 20 & $8.65 \pm 0.03$ & $8.20 \pm 0.03$ & $7.82 \pm 0.03$ \\
\hline \multirow[t]{2}{*}{ RW Aur AB ........... } & 2004 Oct 18 & $8.63 \pm 0.02$ & $8.19 \pm 0.02$ & $7.68 \pm 0.02$ \\
\hline & 2005 Oct 24 & $8.51 \pm 0.02$ & $8.00 \pm 0.02$ & $7.54 \pm 0.02$ \\
\hline V1002 Sco ........... & 2005 Apr 21 & $8.29 \pm 0.02$ & $7.87 \pm 0.02$ & $7.35 \pm 0.02$ \\
\hline AS $206^{*} \ldots \ldots \ldots \ldots$ & 2005 Apr 21 & $9.0 \pm 0.3$ & $8.5 \pm 0.3$ & $7.9 \pm 0.3$ \\
\hline \multirow[t]{7}{*}{ V1331 Cyg* ......... } & 2004 Sep 12 & $10.01 \pm 0.04$ & $9.64 \pm 0.04$ & $8.98 \pm 0.04$ \\
\hline & 2004 Oct 13 & $10.02 \pm 0.04$ & $9.65 \pm 0.04$ & $9.06 \pm 0.04$ \\
\hline & 2004 Oct 23 & $10.03 \pm 0.04$ & $9.57 \pm 0.04$ & $9.00 \pm 0.04$ \\
\hline & 2005 Nov 17 & $10.06 \pm 0.04$ & $9.63 \pm 0.04$ & $9.03 \pm 0.04$ \\
\hline & 2005 Oct 24 & $10.06 \pm 0.04$ & $9.67 \pm 0.04$ & $9.07 \pm 0.04$ \\
\hline & 2005 Oct 25 & $10.01 \pm 0.04$ & $9.64 \pm 0.04$ & $9.04 \pm 0.04$ \\
\hline & 2005 Oct 26 & $9.93 \pm 0.04$ & $9.54 \pm 0.04$ & $8.95 \pm 0.04$ \\
\hline \multirow[t]{2}{*}{ DI Cep* .................... } & 2004 Oct 13 & $9.1 \pm 0.2$ & $8.6 \pm 0.2$ & $8.2 \pm 0.2$ \\
\hline & 2004 Oct 20 & $9.2 \pm 0.2$ & $8.8 \pm 0.2$ & $8.3 \pm 0.2$ \\
\hline \multirow[t]{4}{*}{ 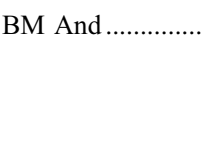 } & 2004 Oct 13 & $9.85 \pm 0.03$ & $9.38 \pm 0.03$ & $8.89 \pm 0.03$ \\
\hline & 2004 Oct 23 & $10.12 \pm 0.03$ & $9.59 \pm 0.03$ & $9.14 \pm 0.03$ \\
\hline & 2005 Oct 24 & $9.61 \pm 0.02$ & $9.10 \pm 0.02$ & $8.61 \pm 0.02$ \\
\hline & 2005 Nov 17 & $9.84 \pm 0.03$ & $9.32 \pm 0.03$ & $8.85 \pm 0.03$ \\
\hline
\end{tabular}

Note.-For AS 206, V1331 Cyg, and DI Cep (indicated by asterisks), our photometric calibrators appear to be variable at a low level, and the quoted uncertainties include this potential error. excess to the normalized, unveiled spectrum, and then renormalizing the sum (e.g., Basri \& Batalha 1990):

$$
F_{\nu, \text { veiled }}=\frac{F_{\nu}+r_{K}}{1+r_{K}} .
$$

For objects observed over multiple epochs, we first fitted $r_{K}$ and $v \sin i \operatorname{simultaneously~for~each~epoch,~fixed~the~} v \sin i$ as the weighted average of values from both epochs (which were consistent within uncertainties in all cases), and then repeated the fits with $v \sin i$ fixed to this average value. Most of the fitted $v \sin i$ values are smaller than the velocity resolution of our observations, $\sim 12 \mathrm{~km} \mathrm{~s}^{-1}$. This means that the $v \sin i$ values of these targets are compatible with those of the WLTT templates. Since the WLTTs used as templates in our veiling determinations may not be slow rotators, their spectra may already be rotationally broadened. Inferred values of $v \sin i$ for our targets thus represent only the amount by which the template spectra need to be further broadened, rather than the true $v \sin i$ of the source, and are only lower limits on the true $v \sin i$ of our sample.

For this fitting, we used $\mathrm{Mg}$ and $\mathrm{Al}$ lines between 2.10 and $2.12 \mu \mathrm{m}$. We restricted the fits to $30 \AA$ regions around strong lines (Fig. 2) to avoid biasing the fits with noisy continuum regions of the spectra. As a check, we applied this fitting procedure to lines of Ti between 2.22 and $2.24 \mu \mathrm{m}$, in a different spectral order than the $\mathrm{Mg}$ and $\mathrm{Al}$ lines; the fitted veilings in both spectral orders agree within $1 \sigma$ uncertainties.

When we fitted veiling to any of our WLTT templates, using the set of remaining WLTTs as templates for the fitting, we found veilings consistent with zero for all objects but one. For TYC 5882, the template with the earliest spectral type, the fitted veiling is $0.2 \pm 0.1$; this is probably due to the mismatch between the spectral type of this and other templates (see Table 1), and does not indicate that TYC 5882 is actually veiled.

There may be some additional uncertainty in the inferred veilings for V1331 Cyg and DI Cep, since these sources have earlier

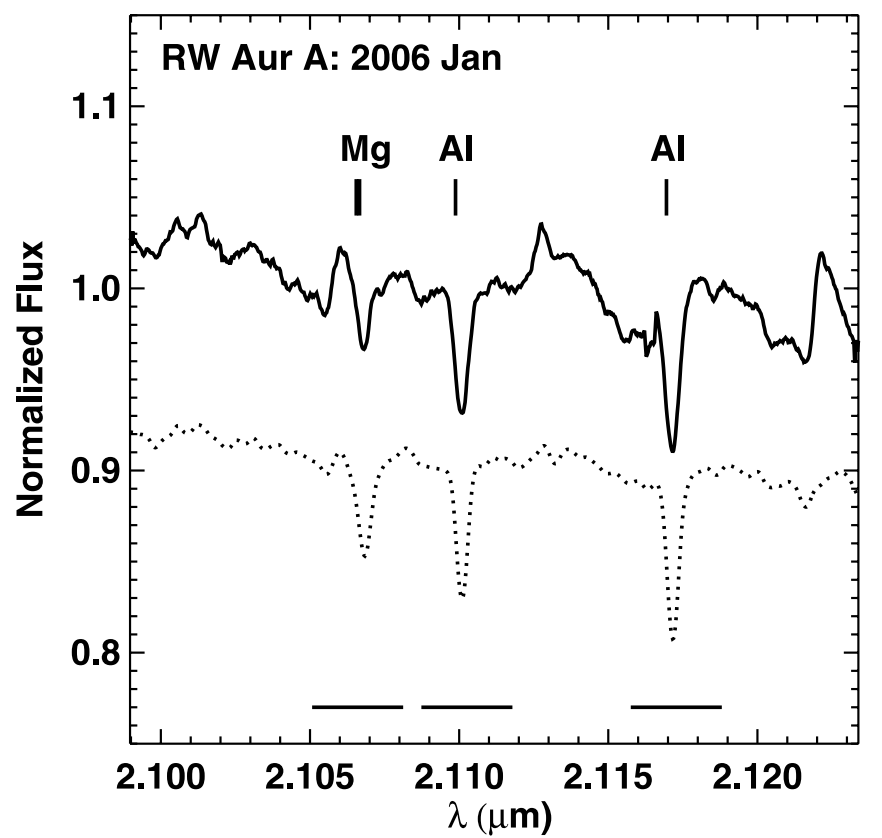

FIG. 2.-Portions of NIRSPEC spectra for RW Aur A (solid curves), with the wavelengths of photospheric Mg and Al absorption lines (e.g., Prato et al. 2003) indicated. The spectrum of a nonaccreting WLTT (AG Tri A), which has been rotationally broadened and veiled (and vertically offset from the target spectrum), is also plotted (dotted curves). Spectral regions used when matching rotationally broadened, veiled template spectra to target spectra are indicated by straight horizontal lines. In 2004 November (left), photospheric absorption lines from $\mathrm{Mg}$ and $\mathrm{Al}$ were significantly more veiled than in 2006 January (right). The inferred veilings are $r_{K}=3.26$ and 1.50 , respectively. We note the presence of emission from the $\mathrm{H}_{2}$ line at $2.12182 \mu \mathrm{m}$ in both epochs; however, analysis of this feature is beyond the scope of this work. 
TABLE 3

$K$-Band Veiling Measurements

\begin{tabular}{|c|c|c|c|c|}
\hline Source & Date & $r_{K}$ & $\begin{array}{l}\text { ional Broadening } \\
\left(\mathrm{km} \mathrm{s}^{-1}\right)\end{array}$ & Template Star \\
\hline CI Tau & 2006 Jan 07 & $1.16 \pm 0.13$ & $<12$ & RX J0437.4A \\
\hline \multirow[t]{2}{*}{ DK Tau A } & 2004 Nov 19 & $1.58 \pm 0.16$ & $<12$ & RX J0437.4B \\
\hline & 2006 Jan 07 & $1.32 \pm 0.14$ & $<12$ & RX J0437.4B \\
\hline \multirow[t]{2}{*}{ DK Tau B } & 2004 Nov 19 & $0.78 \pm 0.10$ & $<12$ & RX J0437.4B \\
\hline & 2006 Jan 07 & $0.80 \pm 0.15$ & $<12$ & RX J0437.4B \\
\hline \multirow[t]{2}{*}{ 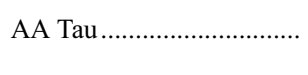 } & 2004 Nov 19 & $0.54 \pm 0.06$ & $<12$ & RX J0437.4B \\
\hline & 2006 Jan 07 & $0.34 \pm 0.06$ & $<12$ & RX J0437.4B \\
\hline \multirow[t]{2}{*}{ RW Aur A } & 2004 Nov 20 & $3.26_{-0.62}^{+0.86}$ & $35 \pm 10$ & AG Tri A \\
\hline & 2006 Jan 07 & $1.50 \pm 0.20$ & $35 \pm 10$ & AG Tri A \\
\hline \multirow[t]{2}{*}{ 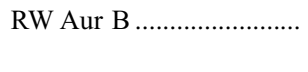 } & 2004 Nov 20 & $0.32 \pm 0.08$ & $11 \pm 4$ & RX J0437.4B \\
\hline & 2006 Jan 07 & $0.28 \pm 0.06$ & $11 \pm 4$ & RX J0437.4B \\
\hline 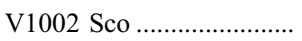 & 2005 Jun 01 & $0.36 \pm 0.08$ & $78 \pm 6$ & RX J1557.8 \\
\hline 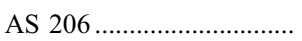 & $2006 \mathrm{Jul} 13$ & $1.48 \pm 0.22$ & $8 \pm 7$ & AG Tri A \\
\hline 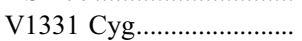 & $2006 \mathrm{Jul} 13$ & $>10$ & $\ldots$ & $\ldots$ \\
\hline 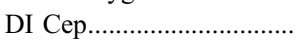 & $2006 \mathrm{Jul} 13$ & $3.40 \pm 0.71$ & $9 \pm 5$ & AG Tri A \\
\hline 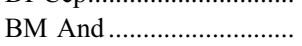 & 2006 Jan 07 & $2.30_{-1.50}^{+0.44}$ & $17 \pm 17$ & TYC 5882 \\
\hline
\end{tabular}

Notes.-The $r_{K}$ and rotational broadening values were determined by minimizing the $\chi^{2}$ residuals between observed spectra and a grid of veiled, rotationally broadened template spectra $(\S 3.1)$. The template stars are WLTTs showing no near-IR excess emission, and fitted veilings for these objects are consistent with zero. However, the templates may have nonnegligible $v \sin i$ values, and thus the fitted rotational broadenings for our targets are lower limits for the true $v \sin i$ values. Quoted error bars are $1 \sigma$ statistical uncertainties in the best-fit parameters. Spectral types of template stars can be found in Table 1 .

spectral types (G5 and G8, respectively) than any of our templates (M2-K3; Table 1). To evaluate the effects of this source/template mismatch, we fitted our veiled, rotationally broadened templates to a nonaccreting, $\sim 30$ Myr old G3/5 star (HIP 9141; Zuckerman $\&$ Song 2004). We found that the best-fit veiling for this star is $\sim 0.5$, and thus the veilings inferred for V1331 Cyg and DI Cep may be biased by this amount. We conservatively treat this bias as an uncertainty, added in quadrature to the statistical uncertainties in the fitted veilings; this additional uncertainty is included in Table 3 .

Our veiling measurements fall within $\sim 1$ month of our KI observations, providing estimates of the circumstellar-to-stellar flux ratios nearly contemporaneous with our interferometric measurements. Moreover, this near coincidence allows a comparison of veiling and the size scale of the circumstellar emission at multiple epochs (although there may be some variability in both quantities over the $\sim 1$ month timescales separating the spectroscopic and interferometric observations; see below). Unfortunately, no measurement of the $K$-band veiling is available contemporaneous with the $2002 \mathrm{KI}$ measurements of Akeson et al. (2005a). These authors estimated a circumstellar-to-stellar flux ratio of 0.6 by assuming that optical photometry traced the stellar photosphere, extrapolating the photosphere to $K$ band and then comparing the extrapolation to the measured $K$-band flux. Since the star may be veiled at optical wavelengths (due to emission from an accretion shock), the true photospheric flux may be lower than assumed, and their estimate of the $K$-band veiling is therefore a lower limit. Nevertheless, we adopt the value of 0.6 here, and assume an uncertainty of $20 \%$.

Inferred veiling values are listed in Table 3. For AA Tau and RW Aur A, the veiling varies (at greater than the estimated $1 \sigma$ uncertainty level) from epoch to epoch. This indicates that the relative fluxes of the stellar and circumstellar components in these systems are changing with time.

\subsection{Inner Disk Sizes}

We now turn to analysis of our interferometric data. To determine the angular extent of the $K$-band emission, we analyze our
KI data in the context of a circularly symmetric uniform ring model (e.g., Eisner et al. 2003). This simple model has been shown (e.g., Eisner et al. 2004) to provide a good description of more complex models of inner disks including puffed-up inner rims (Dullemond et al. 2001; Isella \& Natta 2005). Furthermore, ring models have been used by previous investigators (e.g., MillanGabet et al. 2007), and our use of this model will facilitate comparisons with previous results.

We first remove the contribution to our measured $V^{2}$ from the unresolved central star. As described by Eisner et al. (2005) decomposing the observed $V^{2}$ into stellar and circumstellar components requires a measurement of the circumstellar-to-stellar flux ratio; the veilings determined in $\S 3.1$ provide this ratio for our sample. Contributions from the stellar and circumstellar components add linearly to the measured visibilities, and one can write

$$
V_{\text {disk }}^{2}=\left[\frac{\sqrt{V_{\text {meas }}^{2}}\left(1+F_{\text {disk }} / F_{*}\right)-1}{F_{\text {disk }} / F_{*}}\right]^{2},
$$

where $F_{\text {disk }} / F_{*}$ is the circumstellar-to-stellar flux ratio. The $V_{\text {disk }}^{2}$ values for our sample, obtained using the veiling values measured closest in time to the measured $V^{2}$, are plotted in Figure 3.

Uncertainties in our inferred circumstellar-to-stellar flux ratios lead to uncertainties in $V_{\text {disk }}^{2}$ in addition to those associated with $V_{\text {meas }}^{2}$. This error is smaller when the circumstellar-to-stellar flux ratio is large. Given inferred veilings and associated uncertainties for our sample, the resulting additional uncertainty is less than a few percent. For the first epoch of data from RW Aur A, adopted from Akeson et al. (2005a), the uncertainty is likely to be larger $(\S 3.1)$.

When decomposing observed $V^{2}$ values into stellar and circumstellar parts, each epoch of KI observations was matched to the $K$-band veiling measurement closest in time. Because our interferometric and spectroscopic observations are not exactly simultaneous (generally, measurements are separated by a $\sim 1$ month), and objects may vary photometrically (at both optical and near-IR wavelengths) on this timescale (e.g., Herbst et al. 1994; Skrutskie 

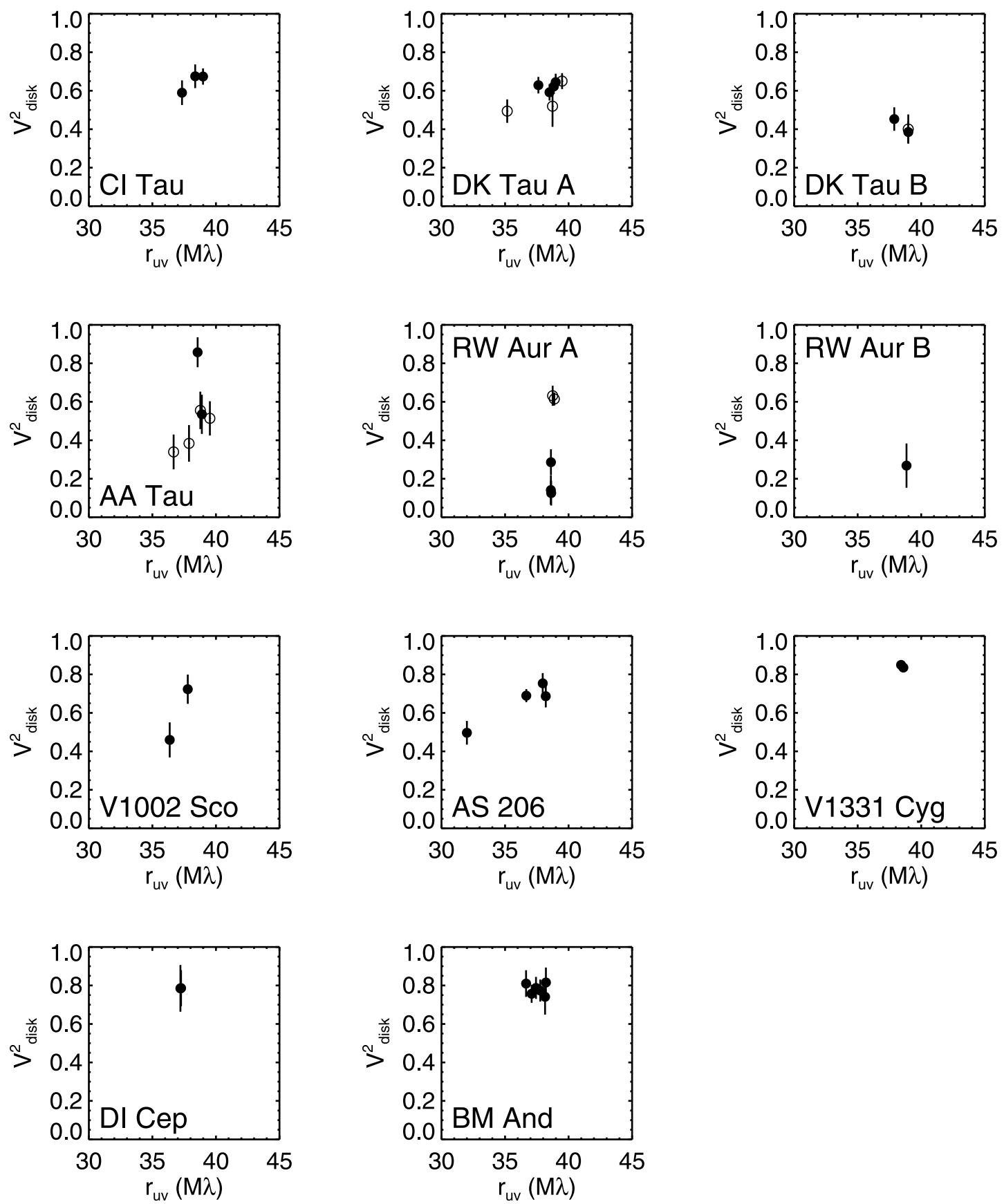

Fig. 3.-Circumstellar component of the measured $V^{2}$ from KI ( $V_{\text {disk }}^{2}$ as computed from eq. [2]). These squared visibilities represent the disk component only. Plotted error bars include uncertainties in measured $V^{2}$ and in circumstellar-to-stellar flux ratios. Symbols and KI data for RW Aur A same as in Fig. 1.

et al. 1996; Eiroa et al. 2002), the inferred circumstellar-to-stellar flux ratios may not represent the true values at the time of the KI observations. Given this potential variability, we also decomposed our multiepoch $V_{\text {meas }}^{2}$ into stellar and circumstellar components by adopting the average value of the veiling over all observed epochs.

The circumstellar components of the visibilities, $V_{\text {disk }}^{2}$, were fitted with uniform ring models using a $\chi^{2}$ minimization to find the model providing the lowest residuals between predicted and measured $V^{2}$. For DK Tau A and B, AA Tau, and RW Aur A, we fitted the ring model to each epoch of our KI observations. Best-fit radii for these face-on uniform ring models, using both the "nearestin-time" and "average value" circumstellar-to-stellar flux ratio determinations, are listed in Table 4. Uncertainties listed in the table are $1 \sigma$ statistical uncertainties in the fits, obtained by computing the surface where $\chi^{2}=\chi_{\min }^{2}+1$. Both approaches to estimating the circumstellar-to-stellar flux ratios for multiepoch observations yield sizes consistent within $1 \sigma$ uncertainties. In the analysis that follows, we will use the sizes derived with the nearest-in-time veiling measurements.

Figure 3 shows that the $V^{2}$ for some objects (AATau, DK Tau A, RW Aur A, V1002 Sco, and AS 206) do not decrease with increasing projected baseline length; these data contrast with the predictions of circularly symmetric disk models. Photometrically determined stellar rotation periods combined with $v \sin i$ measurements suggest highly inclined geometries for some of these sources (e.g., Bouvier et al. 1995), consistent with the nonmonotonic behavior of $V^{2}$ versus baseline length in Figure 3. However, because we are using a single-baseline interferometer 
TABLE 4

FACE-ON RING RADII

\begin{tabular}{|c|c|c|c|}
\hline $\begin{array}{l}\text { Source } \\
\text { (1) }\end{array}$ & $\begin{array}{l}\text { Date } \\
\text { (2) }\end{array}$ & $\begin{array}{c}R_{\text {ring }} \\
\text { (AU) } \\
(3)\end{array}$ & $\begin{array}{c}R_{\text {ring }} \\
\text { (Fixed } r_{K} \text { ) } \\
\text { (4) }\end{array}$ \\
\hline 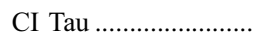 & 2005 Oct 24 & $0.097 \pm 0.008$ & $0.097 \pm 0.008$ \\
\hline \multirow[t]{2}{*}{ DK Tau A .................... } & 2004 Oct 30 & $0.103 \pm 0.005$ & $0.105 \pm 0.005$ \\
\hline & 2005 Oct 24 & $0.107 \pm 0.008$ & $0.105 \pm 0.008$ \\
\hline \multirow[t]{2}{*}{ DK Tau B .................... } & 2004 Oct 30 & $0.137 \pm 0.018$ & $0.137 \pm 0.012$ \\
\hline & 2005 Oct 24 & $0.137 \pm 0.018$ & $0.138 \pm 0.018$ \\
\hline \multirow[t]{2}{*}{ AA Tau ............................ } & 2004 Oct 30 & $0.084 \pm 0.029$ & $0.090 \pm 0.031$ \\
\hline & 2005 Oct 24 & $0.133 \pm 0.019$ & $0.120 \pm 0.016$ \\
\hline \multirow[t]{2}{*}{ RW Aur A ..................... } & 2002 Oct 23 & $0.103 \pm 0.005$ & $0.111 \pm 0.007$ \\
\hline & 2004 Oct 30 & $0.181 \pm 0.016$ & $0.131 \pm 0.007$ \\
\hline RW Aur B ... & 2005 Oct 24 & $0.161 \pm 0.034$ & $0.161 \pm 0.034$ \\
\hline V1002 Sco ..................... & 2005 Apr 21 & $0.118 \pm 0.027$ & $0.118 \pm 0.027$ \\
\hline 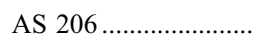 & 2005 Apr 21 & $0.112 \pm 0.007$ & $0.112 \pm 0.007$ \\
\hline V1331 Cyg..................... & 2005 Oct 24 & $0.315 \pm 0.021$ & $0.315 \pm 0.021$ \\
\hline 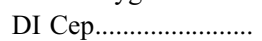 & 2005 Oct 24 & $0.165 \pm 0.039$ & $0.165 \pm 0.039$ \\
\hline BM And ....................... & 2005 Oct 24 & $0.249 \pm 0.028$ & $0.249 \pm 0.028$ \\
\hline
\end{tabular}

Notes.-The $R_{\text {ring }}$ values are computed from fits of uniform ring models to KI data, and the distances assumed in Table 1. Quoted error bars are $1 \sigma$ statistical uncertainties in the $\chi^{2}$ minimization between models and measured $V_{\text {disk }}^{2}$. Col. (3) lists the ring radii determined when $V_{\text {disk }}^{2}$ values are computed using the veiling measurement closest in time to the KI measurement. Col. (4) lists the ring radii for $V_{\text {disk }}^{2}$ estimated using the average veiling measured over all epochs.

(with a fixed separation and position angle), our data generally lack sufficient position angle coverage to place meaningful constraints on inclination (even accounting for Earth rotation, which helps to fill in the position angle coverage).

If we fit our data (assuming that multiple epochs of data trace a nonvariable source) with an inclined uniform disk model (see, e.g., Eisner et al. 2003), we generally cannot rule out an inclination of $0^{\circ}$ (within $1 \sigma$ uncertainties). ${ }^{6}$ Thus, we cannot tell whether the nonmonotonic behavior seen in Figure 3 is due to asymmetric source geometries or uncertainties in the measurements. For one source, RW Aur A, our data are sufficient to constrain the inclination. Assuming the source geometry has not varied over the two epochs of observation (see $\S 4.2$ for a discussion of this assumption), the best-fit inclined disk model has an inclination of $77_{-15}^{+13}$ degrees.

\subsection{Accretion Luminosities}

One of our aims in this analysis is to compare inferred inner disk properties with stellar and accretion properties. Accretion luminosity is a particularly interesting property to correlate with our inner disk measurements, since accretion can provide additional heating that modifies the inner disk structure. Here we use the $\mathrm{Br} \gamma$ emission observed in our NIRSPEC data as a diagnostic of infall (Muzerolle et al. 1998, 2001), and thereby estimate accretion luminosities.

$\mathrm{Br} \gamma$ emission lines for our observed sources are shown in Figure 4 . We measure the $\mathrm{Br} \gamma$ equivalent widths (EW) of our targets from these NIRSPEC observations by (1) determining the spectral baseline by fitting a straight line to a version of the spectrum that is interpolated over the $\mathrm{Br} \gamma$ feature; (2) removing this baseline from the (unsmoothed) target spectrum; and (3) integrating under the $\mathrm{Br} \gamma$ line (over wavelengths from 2.157 to $2.175 \mu \mathrm{m}$ ). We do not need to account for veiling here, since we are ultimately interested in the total line luminosity, which depends on the line EW as well

\footnotetext{
${ }^{6}$ For three-parameter (inner disk radius, position angle, and inclination) fits, $1 \sigma$ uncertainties are computed from projections of the surface with $\chi^{2}=\chi_{\min }^{2}+3.53$.
}

as the continuum level (i.e., the correction to the EW due to veiling would be offset by deveiling the continuum when computing the line flux).

Uncertainties in these EW measurements arise from noise in the spectra and residual errors in calibration due to imperfect interpolation over the broad $\mathrm{Br} \gamma$ absorption features in the telluric standards. We assess these uncertainty sources by applying the same EW measurement procedure to nonaccreting, WLTTs. We find that the $1 \sigma$ uncertainty level for EW estimates is $0.1-0.2 \AA$ for most sources. For one object, V1002 Sco, the calibration did not work as well, and the error is $6 \AA$. EWs and error bars for each source and epoch of observation are listed in Table 5.

We use the $\operatorname{Br} \gamma$ EW measured from our NIRSPEC data and the $K_{s}$-band magnitude determined from our photometry to estimate the $\mathrm{Br} \gamma$ line flux. For close binaries, where our near-IR photometry measures composite fluxes of the system, we estimate fluxes of individual components using previously measured component flux ratios (Simon et al. 1995; White \& Ghez 2001), which involves the implicit assumption that both component fluxes vary if the composite flux varies. We deredden our measured $K_{s}$-band magnitudes using $A_{V}$ values from the literature (White \& Ghez 2001; Eiroa et al. 2002; Walter et al. 1994; Hamann \& Persson 1992) and the reddening law of Steenman \& The (1991) under the assumption that $A_{V}$ is not time variable. We convert these dereddened magnitudes into units offlux density, $F_{\lambda}$. We then compute the flux in the $\mathrm{Br} \gamma$ line by multiplying $F_{\lambda}$ by the EW: $F_{\mathrm{Br} \gamma}=$ $F_{\lambda} \mathrm{EW}$. The $\mathrm{Br} \gamma$ luminosity is then $L_{\mathrm{Br} \gamma}=4 \pi d^{2} F_{\mathrm{Br} \gamma}$, where $d$ is the distance. The $\mathrm{Br} \gamma$ luminosity is related to the accretion luminosity by an empirical relation from Muzerolle et al. (1998, 2001): $\log \left(L_{\mathrm{acc}} / L_{\odot}\right)=(1.26 \pm 0.19) \log \left(L_{\mathrm{Br} \gamma} / L_{\odot}\right)+(4.43 \pm 0.79)$.

Inferred values of $L_{\mathrm{Br} \gamma}$ and $L_{\mathrm{acc}}$ are included in Table 5. Our estimated $L_{\text {acc }}$ values are compatible with previous measurements from the literature (e.g., Valenti et al. 1993; Hartigan et al. 1995; Gullbring et al. 1998; Calvet \& Gullbring 1998; White \& Ghez 2001). Previous measurements typically span an order of magnitude, and our inferred $L_{\text {acc }}$ are within an order of magnitude of all previously determined values. Moreover, the values for each star relative to others in our sample roughly match those of surveys in the literature; in surveys where several of our targets were observed, the lowest accretion rate objects are also the lowest accretors in that subset of our sample.

\section{DISCUSSION}

\subsection{Size-Luminosity Diagram}

If dusty disks extended inward to their central stars, their near-IR emission would be very compact, so much so that it would appear unresolved even with the milliarcsecond level angular resolution of near-IR interferometers. The fact that disks around young stars are resolved with near-IR interferometric data (e.g., Millan-Gabet et al. 1999) indicates that these disks are truncated at stellocentric radii substantially larger than the stellar radius. Measured temperatures at the disk truncation radii are typically $\sim 1500-2000 \mathrm{~K}$ (e.g., Eisner et al. 2004, 2005), compatible with the sublimation temperature of silicate dust (e.g., Pollack et al. 1994). Determining how the inner disk truncation radius depends on source luminosity can constrain how the inner edge is heated and, in turn, the radial and vertical structure of the inner disk (e.g., Monnier \& MillanGabet 2002; Eisner et al. 2004).

Figure 5 shows the inner ring radii determined for a sample of $\mathrm{T}$ Tauri and Herbig Ae/Be objects (including previous measurements from the literature) as a function of source luminosity. The source luminosity is the sum of the stellar luminosity and the accretion luminosity; in cases where no measurement of accretion 

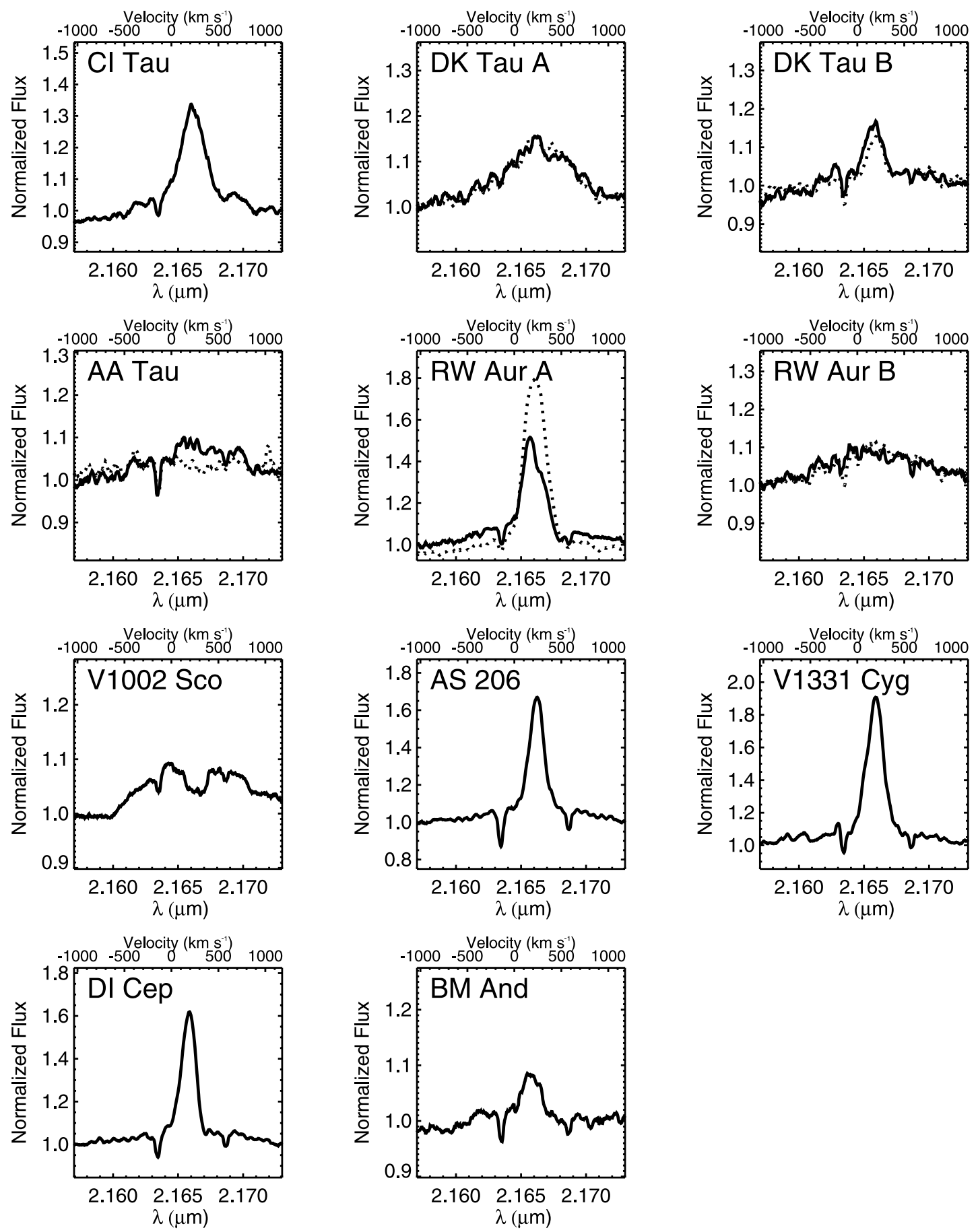

FIG. 4.-Br $\gamma$ lines observed for our sample. For objects observed over multiple epochs, the line observed during the first epoch is plotted with a solid line, and the line observed during the second epoch is represented with a dotted line.

luminosity is available (mostly high-mass stars), only the stellar luminosity is used. Inner ring sizes from the literature are drawn from Millan-Gabet et al. (2001), Eisner et al. (2004, 2005), Akeson et al. (2000, 2005a, 2005b), and Monnier et al. (2005); we used luminosities for these sources quoted in these references. For the objects in our current sample, we took stellar luminosities from Kenyon \& Hartmann (1995), White \& Ghez (2001), Walter et al. (1994), and Hamann \& Persson (1992), and accretion luminosities from Table 5. For objects where we measured the inner disk size over multiple epochs, the average size is plotted in Figure 5.

We compare the size-luminosity diagram in Figure 5 with the predictions of a simple physical model. Specifically, we assume that the inner disk is directly irradiated by the central star, and that the inner edge therefore puffs up (Dullemond et al. 2001). For this model, the location of the inner disk edge is

$$
R_{\mathrm{in}}=\sqrt{(1+f)\left(\frac{L_{*}+L_{\mathrm{acc}}}{4 \pi \sigma T_{\mathrm{in}}^{4}}\right)} .
$$

Here, $f$ is the ratio of the inner edge height to its radius, and is estimated to be between 0.1 and 0.2 for T Tauri and Herbig Ae/Be stars (Dullemond et al. 2001); $T_{\text {in }}$ is the temperature at the inner disk edge. Figure 5 shows the inner disk radii predicted by this 
TABLE 5

$\operatorname{Br} \gamma$ EMISSION Properties

\begin{tabular}{|c|c|c|c|c|c|}
\hline Source & Date & $\begin{array}{l}\text { EW } \\
(\AA)\end{array}$ & $\begin{array}{c}L_{\mathrm{Br} \gamma} \\
\left(10^{-4} L_{\odot}\right)\end{array}$ & $\begin{array}{l}L_{\text {acc }} \\
\left(L_{\odot}\right)\end{array}$ & $\begin{array}{c}L_{*} \\
\left(L_{\odot}\right)\end{array}$ \\
\hline CI Tau ..................... & 2006 Jan 07 & $7.4 \pm 0.1$ & 2.3 & 0.70 & 0.8 \\
\hline \multirow[t]{2}{*}{ DK Tau A ................ } & 2004 Nov 19 & $7.6 \pm 0.1$ & 2.6 & 0.80 & 1.7 \\
\hline & 2006 Jan 07 & $6.9 \pm 0.1$ & 2.5 & 0.76 & 1.7 \\
\hline \multirow[t]{2}{*}{ DK Tau B ................... } & 2004 Nov 19 & $2.7 \pm 0.1$ & 0.3 & 0.05 & 0.5 \\
\hline & 2006 Jan 07 & $1.8 \pm 0.1$ & 0.2 & 0.03 & 0.5 \\
\hline \multirow[t]{2}{*}{ 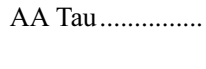 } & 2004 Nov 19 & $4.8 \pm 0.1$ & 0.7 & 0.16 & 0.6 \\
\hline & 2006 Jan 07 & $0.2 \pm 0.1$ & $<0.1$ & $<0.01$ & 0.6 \\
\hline \multirow[t]{2}{*}{ RW Aur A ............. } & 2004 Nov 20 & $9.3 \pm 0.1$ & 1.5 & 0.42 & 1.7 \\
\hline & 2006 Jan 07 & $16.5 \pm 0.1$ & 3.1 & 1.02 & 1.7 \\
\hline \multirow[t]{2}{*}{ 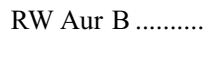 } & 2004 Nov 20 & $6.7 \pm 0.1$ & 0.3 & 0.05 & 0.4 \\
\hline & 2006 Jan 07 & $5.1 \pm 0.1$ & 0.3 & 0.04 & 0.4 \\
\hline V1002 Sco ........... & 2005 Jun 01 & $14 \pm 6$ & 5.4 & 2.07 & 3.8 \\
\hline AS $206 \ldots \ldots \ldots \ldots$ & 2006 Jul 13 & $9.7 \pm 0.2$ & 2.4 & 0.74 & 1.6 \\
\hline V1331 Cyg.......... & $2006 \mathrm{Jul} 13$ & $14.4 \pm 0.2$ & 24.5 & 13.78 & 21.0 \\
\hline DI Cep..................... & $2006 \mathrm{Jul} 13$ & $8.4 \pm 0.2$ & 4.6 & 1.69 & 5.2 \\
\hline 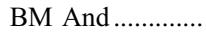 & 2006 Jan 07 & $1.4 \pm 0.1$ & 1.0 & 0.23 & 5.5 \\
\hline
\end{tabular}

Notes.-Given the uncertainties in the photometry and EWs, we estimate uncertainties between $5 \%$ and $20 \%$ for $L_{\mathrm{Br} \gamma}$. The conversion from $L_{\mathrm{Br} \gamma}$ to $L_{\mathrm{acc}}$ includes additional uncertainties (Muzerolle et al. 1998, 2001), and $L_{\text {acc }}$ is thus uncertain by a factor of a few. We also list stellar luminosities, $L_{*}$, drawn from the literature (White \& Ghez 2001; Hartigan et al. 1995; Walter et al. 1994; Hamann \& Persson 1992).

model for $T_{\text {in }}=1500$ and $2000 \mathrm{~K}$; these temperatures bracket the expected sublimation temperatures for silicate dust in a protoplanetary disk (e.g., Pollack et al. 1994).

Most objects with luminosities $\gtrsim 10 L_{\odot}$ agree with the predictions of these models; this fact has been noted previously (Monnier \& Millan-Gabet 2002; Eisner et al. 2004; MillanGabet et al. 2007). There are, however, several exceptional highluminosity sources for which geometrically thin disk models may be more suitable (e.g., Eisner et al. 2004; Monnier \& MillanGabet 2002; Vinković \& Jurkić 2007). Moreover, Figure 5 shows that at the lowest stellar masses, this model may break down. The sizes of several low-mass sources are larger than predicted for these puffed-up inner disk models.

We put forward several potential explanations for these deviations between puffed-up disk model predictions and data for lowmass T Tauri stars. First, the sublimation temperature may be reached farther from the star. For example, viscous energy dissipation in accreting material may provide additional heating, pushing the sublimation radius outward. The sublimation temperature may also be lower, and hence located at larger stellocentric radii, in these low-mass objects because of systematically lower ambient gas densities (see Table 3 of Pollack et al. 1994). Small dust grains, which are hotter than larger grains at a given radius, may also lead to larger dust sublimation radii. Finally, these disks may be truncated by a mechanism other than dust sublimation, such as photoevaporative mass loss or magnetospheric truncation. In the following paragraphs, we argue that of the possible explanations considered, the most likely is a mechanism other than dust sublimation, namely, magnetospheric truncation.

These various possible explanations make different predictions about the accretion rates of sources with exceptionally large inner disk radii. We therefore investigate how the discrepancy between measured sizes and predictions of irradiated disk models depends on a proxy of source accretion rate. ${ }^{7}$ Figure 6 plots the difference

\footnotetext{
${ }^{7}$ We use $L_{\text {acc }}$ rather than $\dot{M}$, since $L_{\text {acc }}$ is closer to the observed quantities. Conversion to $\dot{M}$ requires additional assumptions about stellar mass and radius, and the inner disk radius (e.g., Gullbring et al. 1998).
}

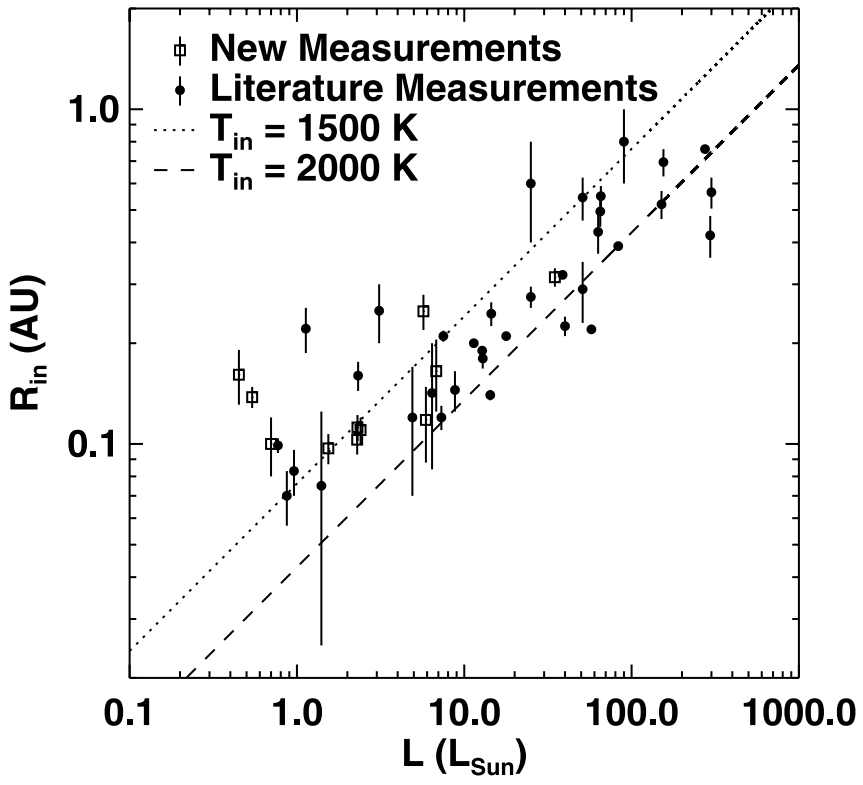

FIG. 5.- Inner ring radii determined from near-IR interferometry, as a function of source luminosity (stellar + accretion luminosities, where available). Inner ring sizes from the literature (circles) and for the sources in our sample (squares) are plotted. We indicate the inner ring radii expected for a puffed-up inner disk model (Dullemond et al. 2001) with $T_{\text {in }}=1500 \mathrm{~K}$ (dotted line) and $T_{\text {in }}=2000 \mathrm{~K}$ (dashed line). While most objects with luminosities $\gtrsim 10 L_{\odot}$ agree with the predictions of these models (with the exception of several high-mass stars whose disks appear consistent with geometrically thin disk models; Eisner et al. 2004), at lower luminosities, there are numerous sources with inner ring radii larger than predicted values.

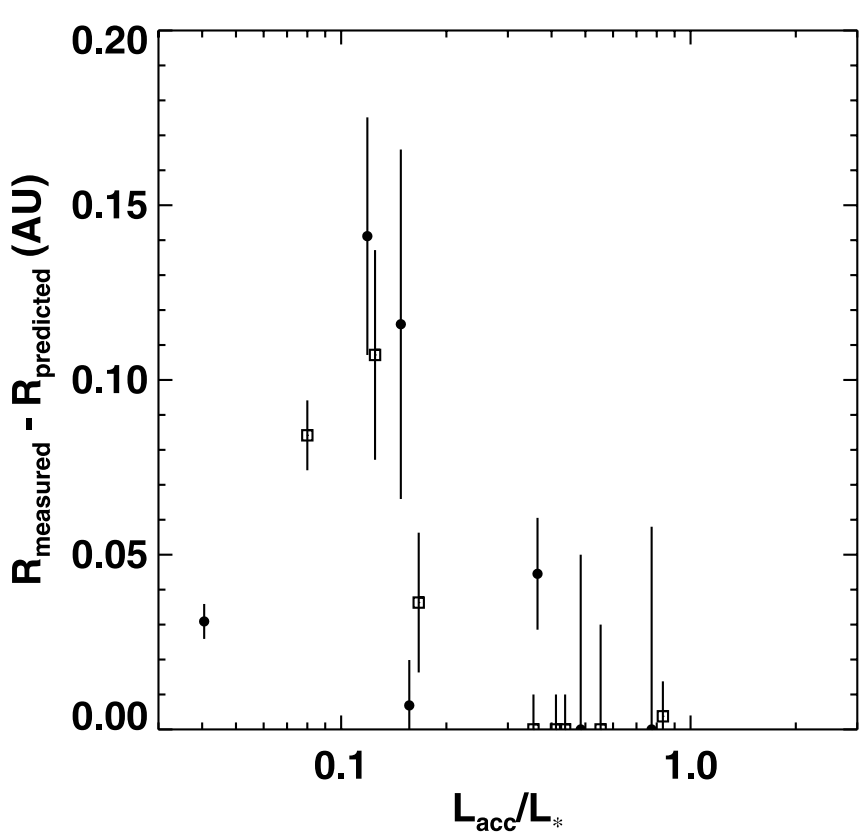

FIG. 6.-Discrepancy between the measured size and the model prediction (in the range of inner disk temperatures between 1500 and $2000 \mathrm{~K}$ ) plotted against the ratio of accretion to stellar luminosity. The discrepancy is the difference between the measured size and the region bounded by the two curves in Fig. 5. As in Fig. 5, discrepancies for sources from the literature are indicated by circles, and for the sources in our sample by squares. We have restricted this plot to T Tauri stars $\left(L_{*}<5 L_{\odot}\right)$, since most higher luminosity objects do not have measured $L_{\text {acc }}$. While some low accretion luminosity sources do agree with the model predictions, a trend is nevertheless evident whereby objects with smaller $L_{\text {acc }} / L_{*}$ ratios are more discrepant from model predictions. The objects exhibiting the largest discrepancies are DK Tau B and RW Aur B from our sample, GM Aur from Akeson et al. (2005a), and AS 207 A from Eisner et al. (2005). 
between measured and predicted size as a function of $L_{\text {acc }} / L_{*}$. We find that objects whose measured sizes are larger than predicted by models actually have lower (fractional) accretion luminosities than other sources.

\subsubsection{Accretional Heating}

If accretional heating causes larger inner disk truncation radii, then the low-mass stars in Figure 5 would have systematically higher mass accretion rates than other sources. However, Figure 6 shows that objects with large inner radii tend to have lower accretion rates. Thus, accretional heating seems an unlikely explanation for the large inner disk sizes.

\subsubsection{Lower Dust Sublimation Temperatures}

Dust sublimates more easily when ambient pressures are lower. Lower mass accretion rates lead to lower gas densities and pressures, and can therefore yield lower sublimation temperatures. The fact that larger disk truncation radii tend to occur around sources with lower accretion luminosities (Fig. 6) is compatible with the hypothesis that these disks are truncated at lower dust sublimation temperatures.

However, the dust sublimation temperature would need to be $\lesssim 1000 \mathrm{~K}$ to explain many of the large measured disk sizes. A silicate dust sublimation temperature of $1000 \mathrm{~K}$ requires 8 orders of magnitude lower gas density than a sublimation temperature of $1500 \mathrm{~K}$ (Pollack et al. 1994), which corresponds to $\sim 5$ orders of magnitude lower mass accretion rates (assuming an $\alpha$-disk to convert gas density into mass accretion rate). Such low accretion rates, and large dispersion in accretion rate, are not measured for our sample. It is therefore unlikely that lower sublimation temperature can explain the large truncation radii of disks around low-mass $\mathrm{T}$ Tauri stars.

\subsubsection{Small Dust Grains}

Smaller dust grains absorb and emit radiation less efficiently than larger grains, and this loss of efficiency is more severe at longer wavelengths (e.g., Bohren \& Huffman 1983). As a result, small grains will achieve higher temperatures than larger grains at a given stellocentric radius. Thus, if a disk is composed of small grains, the dust sublimation radius can be larger than implied by equation (3), which assumed gray, and hence large-grained, dust (Monnier \& Millan-Gabet 2002; Isella et al. 2006; Vinković et al. 2006; Tannirkulam et al. 2007). Using the results of Vinković et al. (2006) we find that the large inner disk sizes in our sample can be reproduced by puffed-up inner disk models composed of grains sized $0.1-0.5 \mu \mathrm{m}$. While small dust grain models can reproduce these larger observed sizes, it seems unlikely that those objects that would require small dust grains to explain the observations are precisely those with the lowest mass accretion rates.

\subsubsection{Photoevaporation}

We now consider alternatives to dust sublimation, where the physical process responsible for truncating the disk might be more efficient at lower mass accretion rates. One such process is photoevaporation, where the mass-loss driven by stellar UV radiation can overcome accretion at sufficiently low $\dot{M}$ (e.g., Clarke et al. 2001; Alexander et al. 2006). The mass flow can "switch" from accretion to outflow when the accretion rate drops below $\sim 10^{-10} M_{\odot} \mathrm{yr}^{-1}$. Since the objects in our sample have accretion rates $\gtrsim 10^{-9} M_{\odot} \mathrm{yr}^{-1}$ (based on the accretion luminosities in Table 5), photoevaporation is unlikely to dominate accretion. Moreover, we do not see the signature of a discrete transition in Figure 6 at a certain accretion luminosity. Thus, photoevapora- tion is unlikely to be the physical mechanism behind the truncated disks observed for the low-mass T Tauri stars in our sample.

\subsubsection{Magnetospheric Truncation}

Large inner disk radii may also be due to magnetospheric truncation. T Tauri stars accrete material via magnetospheric accretion; the stellar magnetic field threads the disk and essentially creates a barrier to midplane accretion at the point of balance between magnetic pressure and inward pressure from accretion (e.g., Shu et al. 1994). The magnetospheric radius is given by (Königl 1991)

$$
\frac{R_{\mathrm{mag}}}{R_{*}}=2.27\left[\frac{\left(B_{0} / 1 \mathrm{kG}\right)^{4}\left(R_{*} / R_{\odot}\right)^{5}}{\left(M_{*} / M_{\odot}\right)\left(\dot{M} / 10^{-7} M_{\odot} \mathrm{yr}^{-1}\right)^{2}}\right]^{1 / 7},
$$

where $B_{0}$ is the dipole magnetic field strength. For many classical T Tauri stars, high disk accretion rates $\left(\gtrsim 10^{-7} M_{\odot} \mathrm{yr}^{-1}\right)$ confine this magnetospheric radius to well within the dust sublimation radius (e.g., Eisner et al. 2005), and thus the observed inner edge of the dust disk occurs where temperatures reach the sublimation temperature (i.e., $T_{\text {in }}=1500-2000 \mathrm{~K}$ ).

However, lower accretion rates allow the magnetospheric radius to extend to larger stellocentric radii $\left(R_{\text {mag }} \propto \dot{M}^{-2 / 7}\right)$, while the lower associated accretion luminosity leads to a smaller stellocentric radius of dust sublimation. Low accretion rates can therefore cause magnetospheric truncation outside of the dust sublimation radius (e.g., Eisner et al. 2006). If we take $B_{0}=1 \mathrm{kG}$, $R_{*}=2 R_{\odot}$, and $M_{*}=0.5 M_{\odot}$ as typical values for low-mass T Tauri stars (e.g., Johns-Krull 2007), then mass accretion rates between $10^{-9}$ and $10^{-8} M_{\odot} \mathrm{yr}^{-1}$ (compatible with the lowest accretion luminosities in Table 5) yield magnetospheric radii between 0.07 and $0.15 \mathrm{AU}$, comparable to the measured inner disk sizes in Figure 5. We thus favor magnetospheric truncation as the explanation for the large inner disk sizes of low-mass T Tauri stars.

\subsection{Inner Disk Variability}

Many young stars, including those in our sample, are known to be photometrically variable at optical through near-IR wavelengths, with variability timescales ranging from days to months (e.g., Herbst et al. 1994; Skrutskie et al. 1996; Carpenter et al. 2001; Eiroa et al. 2002; Bouvier et al. 2007). While the underlying cause of the variability is usually attributed to starspots, which modulate the emission on the stellar rotation period, in some cases inner disk variability may also play a role (e.g., Eiroa et al. 2002; Bouvier et al. 2007).

Time-varying increases in disk accretion rates, which might occur on inner disk dynamical timescales of days to weeks, could lead to increased disk heating and higher near-IR emission. Since a hotter disk could sublimate dust at larger stellocentric radii than a cooler disk, the inner edge of the dust disk may move in and out as the disk heating varies. Conversely, if disks are magnetospherically truncated, then increases in accretion rate would lead to smaller disk sizes (eq. [4]). Variability in the stellar magnetic field strength can also cause variability in mass accretion rates and changes in the inner disk radius over timescales comparable to stellar dynamo oscillation period, typically $\sim 10 \mathrm{yr}$ (e.g., Armitage 1995).

We obtained multiple epochs of spectroscopic and photometric data for DK Tau A and B, AA Tau, and RW Aur A and B, and multiple epochs of interferometric data for all of these except RW Aur B. As indicated in Tables 2-5, several sources have variable infrared fluxes, veilings, and inferred accretion luminosities. Moreover, one 
object (RW Aur A) may have a variable inner disk size (Fig. 3; Table 4).

In the scenario where observed variability is due to changes in the disk heating (e.g., because of time-variable disk accretion rates), we would expect the following for higher disk temperatures: (1) $K$-band flux will increase; (2) veiling will increase, since the circumstellar flux is increasing relative to the stellar flux; (3) accretion luminosity may increase (if this is the source of the disk heating); and (4) the measured inner disk size should increase, since the dust sublimation radius would move out.

None of our targets clearly show such signatures. For DK Tau A, the $K$-band veiling, near-IR fluxes, and inner disk size remain constant (within uncertainties) over the span of our multiepoch observations. For AA Tau, the veiling appears to decrease from one epoch to the next while the $\mathrm{Br} \gamma$ flux also decreases. However, the inner disk size remains constant (or marginally increases; Table 4), and the $K_{s}$-band flux appears to increase in the latter epoch, contrary to expectations for a dimmer disk. For RW Aur A, the $\mathrm{Br} \gamma$ luminosity appears to increase as the veiling decreases, which is not consistent with expectations for our scenario of inner disk variability above. DK Tau B and RW Aur B show no variability in any of our data.

If the emission from our targets is circularly symmetric, then our modeling indicates potential variability (above the $1 \sigma$ level) in the inner disk size with time for RW Aur A. The inner disk radius appears to increase as the $K$-band veiling increases, which is expected, since a larger inner rim would produce more near-IR emission (for a fixed temperature). However, Figure 1 shows only marginal variability in the measured $V^{2}$ (in contrast to the $V_{\text {disk }}^{2}$ plotted in Fig. 3) from one epoch to the next, and the apparent inner disk variability rests entirely on the inferred circumstellarto-stellar flux ratios. The first epoch of KI data for RW Aur A does not have a reliable estimate of this ratio; the estimate of 0.6 from Akeson et al. (2005a) is a lower limit ( $\S 3.1)$, and a ratio as high as 2.5 would eliminate the apparent variability. Furthermore, a highly inclined disk can fit the data for RW Aur A well without any variability in the inner disk size, even if the inferred circumstellar-tostellar flux ratios are taken at face value ( $\S 3.2)$. Thus, we cannot claim to see inner disk variability based on these data.

The interpretation of our multiepoch observations is complicated by the fact that different data constituting each epoch were not obtained simultaneously. Data for a given epoch were obtained within a few weeks time window. Because the dynamical timescales for inner disk variability are $\tau_{\text {dyn }} \sim\left(R^{3} / G M\right)^{1 / 2} \lesssim 10$ days for the inner radii inferred for our sample, there may be substantial source variability between the different measurements within a single epoch. In future work, the observations constituting a single epoch should ideally be obtained within $\sim 1$ day of one another. In addition, a larger number of epochs would facilitate investigation of inner disk variability.

\section{CONCLUSIONS}

Using near-IR interferometric, spectroscopic, and photometric data, we measured the inner disk radii of 11 low-mass T Tauri stars. In addition, we measured the near-IR veilings and accretion luminosities of these objects. Our data substantiate previous claims that the inner disk radii of $\mathrm{T}$ Tauri stars are generally consistent with the predictions of disk models with vertically extended inner rims at stellocentric radii where disk temperatures reach dust sublimation temperatures, between 1500 and 2000 K. However, there are a handful of sources, in particular objects with stellar luminosities $\lesssim 1 L_{\odot}$, for which the measured radii are substantially larger than predicted by such models.

Discrepancies between models where disks are truncated at the dust sublimation radius and measured inner disk radii are larger for sources with low ratios of accretion to stellar luminosity. Thus, the physical mechanism by which the inner disk is truncated appears to depend on the relative importance of stellar irradiation and accretion. To explain this observation, we suggest that at low accretion rates, as the pressure of accreting material drops, the point of balance between inward accretion pressure and outward stellar magnetic pressure moves to larger stellocentric radii. At sufficiently low accretion rates, magnetospheric radii can become larger than the dust sublimation radii, yielding the large observed sizes for low accretion rate objects in our sample.

For several of our sample objects, we obtained multiple epochs of interferometric, spectroscopic, and photometric data, and for each epoch the various data sets were obtained within a few weeks of one another. We used these multiepoch observations to search for inner disk variability. While inferred veilings, accretion luminosities, and even inner disk truncation radii appear to vary for some sources, none of the objects show a variability signature consistent with changes in the inner disk. Future monitoring observations with more epochs, and with different data comprising each epoch obtained within a time window short compared to inner disk dynamical timescales, will enable a more rigorous probe of inner disk variability.

The near-IR interferometry data presented in this paper were obtained with the Keck Interferometer (KI), which is supported by NASA. We wish to thank the entire KI team for making these observations possible. KI and NIRSPEC observations were carried out at the W. M. Keck Observatory, which is operated as a scientific partnership among California Institute of Technology, the University of California, and NASA. The Observatory was made possible by the generous financial support of the W. M. Keck Foundation. The authors wish to recognize and acknowledge the cultural role and reverence that the summit of Mauna Kea has always had within the indigenous Hawaiian community. We are most fortunate to have the opportunity to conduct observations from this mountain. This work has used software from the Michelson Science Center at the California Institute of Technology. J. A. E. acknowledges support from a Miller Research Fellowship, and thanks G. Basri for useful discussions and input into various stages of this work. J. A. E. is also grateful to F. Ciesla for interesting discussions about dust sublimation temperatures, and to E. Lopez for his interest in this project.

\section{REFERENCES}

Adams, F. C., Lada, C. J., \& Shu, F. H. 1988, ApJ, 326, 865

Akeson, R. L., Ciardi, D. R., van Belle, G. T., Creech-Eakman, M. J., \& Lada,

E. A. 2000, ApJ, 543, 313

Akeson, R. L., et al. 2005a, ApJ, 635, 1173

2005b, ApJ, 622, 440

Alexander, R. D., Clarke, C. J., \& Pringle, J. E. 2006, MNRAS, 369, 229

Armitage, P. J. 1995, MNRAS, 274, 1242

Aveni, A. F., \& Hunter, J. H. 1969, AJ, 74, 1021

Basri, G., \& Batalha, C. 1990, ApJ, 363, 654
Basri, G., \& Bertout, C. 1989, ApJ, 341, 340

Beckwith, S. V. W., Sargent, A. I., Chini, R. S., \& Guesten, R. 1990, AJ, 99, 924

Bertout, C., Basri, G., \& Bouvier, J. 1988, ApJ, 330, 350

Bloom, J. S., Starr, D. L., Blake, C. H., Skrutskie, M. F., \& Falco, E. E. 2006, in ASP Conf. Ser. 351, Astronomical Data Analysis Software and Systems XV, ed. C. Gabriel et al. (San Francisco: ASP), 751

Boden, A. F., Colavita, M. M., van Belle, G. T., \& Shao, M. 1998, Proc. SPIE, 3350,872 
Bohren, C. F., \& Huffman, D. R. 1983, Absorption and Scattering of Light by Small Particles (New York: Wiley)

Bouvier, J., Covino, E., Kovo, O., Martin, E. L., Matthews, J. M., Terranegra, L., \& Beck, S. C. 1995, A\&A, 299, 89

Bouvier, J., et al. 2007, A\&A, 463, 1017

Calvet, N., \& Gullbring, E. 1998, ApJ, 509, 802

Carpenter, J. M., Hillenbrand, L. A., \& Skrutskie, M. F. 2001, AJ, 121, 3160

Chavarria-K., C. 1981, A\&A, 101, 105

Clarke, C. J., Gendrin, A., \& Sotomayor, M. 2001, MNRAS, 328, 485

Colavita, M. M. 1999, PASP, 111, 111

Colavita, M. M., \& Wizinowich, P. L. 2003, Proc. SPIE, 4838, 79

Colavita, M. M., et al. 2003, ApJ, 592, L83

Dullemond, C. P., Dominik, C., \& Natta, A. 2001, ApJ, 560, 957

Dutrey, A., Guilloteau, S., Duvert, G., Prato, L., Simon, M., Schuster, K., \& Menard, F. 1996, A\&A, 309, 493

Edwards, S., Hartigan, P., Ghandour, L., \& Andrulis, C. 1994, AJ, 108, 1056

Eiroa, C., et al. 2002, A\&A, 384, 1038

Eisner, J. A., Chiang, E. I., \& Hillenbrand, L. A. 2006, ApJ, 637, L133

Eisner, J. A., Chiang, E. I., Lane, B. F., \& Akeson, R. L. 2007, ApJ, 657, 347

Eisner, J. A., Hillenbrand, L. A., White, R. J., Akeson, R. L., \& Sargent, A. I. 2005, ApJ, 623, 952

Eisner, J. A., Lane, B. F., Akeson, R. L., Hillenbrand, L., \& Sargent, A. 2003, ApJ, 588, 360

Eisner, J. A., Lane, B. F., Hillenbrand, L., Akeson, R., \& Sargent, A. 2004 ApJ, 613, 1049

Gray, D. F. 1992, The Observation and Analysis of Stellar Photospheres (2nd ed.; Cambridge: Cambridge Univ. Press)

Gullbring, E., Hartmann, L., Briceno, C., \& Calvet, N. 1998, ApJ, 492, 323

Hamann, F., \& Persson, S. E. 1992, ApJ, 394, 628

Hartigan, P., Edwards, S., \& Ghandour, L. 1995, ApJ, 452, 736

Hayashi, C. 1981, Prog. Theor. Phys., 70, 35

Herbig, G. H., \& Bell, K. R. 1988, Third Catalog of Emission Line Stars of the Orion Population (Santa Cruz: Lick Obs.)

Herbst, W., Herbst, D. K., Grossman, E. J., \& Weinstein, D. 1994, AJ, 108, 1906

Isella, A., \& Natta, A. 2005, A\&A, 438, 899

Isella, A., Testi, L., \& Natta, A. 2006, A\&A, 451, 951

Johns-Krull, C. M. 2007, ApJ, 664, 975

Kenyon, S. J., \& Hartmann, L. 1995, ApJS, 101, 117

Kholopov, P. N. 1959, Soviet Astron., 3, 291

Koerner, D. W., \& Sargent, A. I. 1995, AJ, 109, 2138

Köhler, R., Kunkel, M., Leinert, C., \& Zinnecker, H. 2000, A\&A, 356, 541

Königl, A. 1991, ApJ, 370, L39
Leinert, C., et al. 2004, A\&A, 423, 537

Lin, D. N. C., Bodenheimer, P., \& Richardson, D. C. 1996, Nature, 380, 606

Liu, W. M., Hinz, P. M., Meyer, M. R., Mamajek, E. E., Hoffmann, W. F., Brusa, G., Miller, D., \& Kenworthy, M. A. 2007, ApJ, 658, 1164

Marcy, G., Butler, R. P., Fischer, D., Vogt, S., Wright, J. T., Tinney, C. G., \& Jones, H. R. A. 2005, Prog. Theor. Phys. Suppl., 158, 24

McCaughrean, M. J., \& O’Dell, C. R. 1996, AJ, 111, 1977

McLean, I. S., McGovern, M. R., Burgasser, A. J., Kirkpatrick, J. D., Prato, L., \& Kim, S. S. 2003, ApJ, 596, 561

Millan-Gabet, R., Malbet, F., Akeson, R., Leinert, C., Monnier, J., \& Waters, R. 2007, in Protostars and Planets V, ed. B. Reipurth, D. Jewitt, \& K. Keil (Tucson: Univ. Arizona Press), 539

Millan-Gabet, R., Schloerb, F. P., \& Traub, W. A. 2001, ApJ, 546, 358

Millan-Gabet, R., Schloerb, F. P., Traub, W. A., Malbet, F., Berger, J. P., \& Bregman, J. D. 1999, ApJ, 513, L131

Monin, J.-L., Menard, F., \& Duchene, G. 1998, A\&A, 339, 113

Monnier, J. D., \& Millan-Gabet, R. 2002, ApJ, 579, 694

Monnier, J. D., et al. 2005, ApJ, 624, 832 2006, ApJ, 647, 444

Mora, A., et al. 2001, A\&A, 378, 116

Muzerolle, J., Calvet, N., \& Hartmann, L. 2001, ApJ, 550, 944

Muzerolle, J., Hartmann, L., \& Calvet, N. 1998, AJ, 116, 2965

Perryman, M. A. C., et al. 1997, A\&A, 323, L49

Pollack, J. B., Hollenbach, D., Beckwith, S., Simonelli, D. P., Roush, T., \& Fong, W. 1994, ApJ, 421, 615

Prato, L., Greene, T. P., \& Simon, M. 2003, ApJ, 584, 853

Shu, F., Najita, J., Ostriker, E., Wilkin, F., Ruden, S., \& Lizano, S. 1994, ApJ, 429,781

Simon, M., et al. 1995, ApJ, 443, 625

Skrutskie, M. F., Meyer, M. R., Whalen, D., \& Hamilton, C. 1996, AJ, 112, 2168

Steenman, H., \& Thé, P. S. 1991, Ap\&SS, 184, 9

Tannirkulam, A., Harries, T. J., \& Monnier, J. D. 2007, ApJ, 661, 374

Valenti, J. A., Basri, G., \& Johns, C. M. 1993, AJ, 106, 2024

van Boekel, R., et al. 2004, Nature, 432, 479

Vinković, D., Ivezić, Ž., Jurkić, T., \& Elitzur, M. 2006, ApJ, 636, 348

Vinković, D., \& Jurkić, T. 2007, ApJ, 658, 462

Walker, M. F. 1972, ApJ, 175, 89

Walter, F. M., Vrba, F. J., Mathieu, R. D., Brown, A., \& Myers, P. C. 1994, AJ, 107,692

White, R. J., \& Ghez, A. M. 2001, ApJ, 556, 265

Wichmann, R., et al. 2000, A\&A, 359, 181

Zuckerman, B., \& Song, I. 2004, ARA\&A, 42, 685 